\title{
Polypyrrole/Sisal Fiber Composites for Energy Storage
}

\author{
H.D. Mo, C. Yang, C. Wei, F.A. Zhang, S.R. Lu, Z.Q. \\ Wang, X.X. Huang \\ State Key Laboratory Breeding Base of Nonferrous Metals \& \\ Specific Materials Processing; Key Laboratory of New \\ Processing Technology for Nonferrous Metals \& Materials \\ Guilin University of Technology \\ Guilin, China
}

\author{
L.M. Zang \\ Department of Machine Intelligence and Systems Engineering, \\ Faculty of Systems Engineering \\ Akita Prefectural University \\ Akita, Japan
}

\begin{abstract}
Polypyrrole-based electrodes were fabricated for energy storage applications using sisal fibers as a substrate material. It was found that the PPy nanoparticles deposited on the surface of sisal fiber cellulose (SFC) connected to form a continuous sheath by taking along the microcrystalline cellulose template. The as-prepared nanocomposites demonstrated a mass-specific capacitance of $367 \mathrm{~F} / \mathrm{g}$ at $200 \mathrm{~mA} / \mathrm{g}$ current density in supercapacitor application. Moreover, SFC/PPy electrode retained about $57.8 \%$ of $C s$ retained when the current density increased five times. This work provides a straightforward method to utilize renewable resource sisal microcrystalline cellulose for conducting composite, which could apply in sensors, flexible electrodes, and flexible displays. It also opens a new field of potential applications of micrometre-scale natural microcrystalline cellulose.
\end{abstract}

Keywords-cellulose; Conducting polymer; Supercapacitor; Renewable resource; Hemp

\section{INTRODUCTION}

Natural fibrous substances play a key role in worldwide modern industries [1]. The especial morphologies of the natural substances afforded gave superior properties in composites. Cellulose supported conducting polymer has received growing interest in recent years due to their better performance or new properties, which has largely potential applications such as batteries, sensors, electrical devices, etc. Polypyrrole (PPy) is one of the most widely investigated conducting polymers because of its excellent thermal and environmental stability and high electrical conductivity [2]. Unfortunately, the poor processability and inadequate mechanical properties of PPy limit its commercial application [2]. In order to overcome these problems, deposition of PPy on fiber surface of fabrics and yarn, such as cotton, silk, and cellulose derivatives have been widely investigated in the last few years. As a especial kind of cellulose, sisal microcrystalline cellulose is produced by sisal hemp. Sisal fiber is one of the most common nature fibers that is the most largely quantity and the most widely used [3-5]. Sisal is a renewable resource par excellence and can form part of the overall solution to climate change. Sisal fiber (SFC) exhibits an ultrafine fibrous network, highly crystalline structure, purity, low density and remarkable mechanical strength. Meanwhile, the raw material was easily obtained. In this view, electrically conducting composed of sisal fiber and polypyrrole that presents a successful combination of the inherent properties of each single component. These properties include high tensile strength, toughness, biocompatibility, high surface areas of the SFC and cytocompatibility, electronic and chemical properties of PPy.

In this paper, we prepared the SFC/PPy nanocomposite. The as-prepared nanocomposites were characterized by FTIR, SEM, and electrochemistry techniques. The SFC/PPy nanocomposites were further evaluated for their applicability for electrochemical energy storage application, and a high special capacitance was obtained.

\section{EXPERIMENTAL SECTION}

\section{A. Materials}

Sisal fibers were provided by Guangxi Sisal Group Co., Ltd., China. Pyrrole, ammonium persulfate, sodium p-toluene sulfonate, sodium tetraborate, acetic acid, and nitric acid were purchased from Nacalai Tesque, Inc., Japan. Pyrrole monomer dehydrated with calcium hydride for $24 \mathrm{~h}$ was distilled under reduced pressure before use. All other chemical reagents were in analytical grade. All solutions were prepared in deionized water.

\section{B. Preparation}

The sisal fiber cellulose (SFC) was produced from sisal fibers as reported [2]. The SFC/PPy nanocomposite was prepared via in situ oxidative polymerization-induced adsorption onto SFC micro-rods. A typical synthesis was as follows: STS (4.16 g) was dissolved in $100 \mathrm{ml}$ of deionized water, and then a certain amount of SFC were added $(50.9 \mathrm{mg}$, $107.4 \mathrm{mg}, 241.8 \mathrm{mg}, 414.4 \mathrm{mg}$, and $967.0 \mathrm{mg}$; named as: S1, S2, S3, S4, and S5, respectively). The mixture was then ultrasonically dispersed, and pyrrole $(1 \mathrm{ml})$ was added into the mixture with vigorous stirring. Afterward, the mixture was mechanically stirred for $30 \mathrm{~min}$ at $0{ }^{\circ} \mathrm{C}$. Then an aqueous solution $(20 \mathrm{ml})$ of APS $(0.90 \mathrm{~g})$ was added drop by drop to the above mixture instantly to start the oxidative polymerization. The reaction was performed under mechanical stirring for $8 \mathrm{~h}$. The resulting precipitates were washed with deionized water and ethanol several times. Finally, the product was dried in vacuum at $60^{\circ} \mathrm{C}$ for $24 \mathrm{~h}$ to obtain of the product as a dark powder. 


\section{Measurements}

The morphology and microstructure of the samples were investigated with a S-4800 field emission scanning electron microscope (SEM; HITACHI, Japan). The Fourier transform infrared spectroscopy (FTIR) measurements (Impact 400, Nicolet, Waltham, MA) were carried out with the KBr pellet method. Electrochemical experiments were performed on an electrochemical workstation (CHI660D, China) with a conventional three-electrode system. The working electrode was prepared by mixing the active material with $15 \mathrm{wt} \%$ acetylene black and $5 \mathrm{wt} \%$ Polyvinylidene fluoride (based on the total electrode mass) to form a slurry. Then the slurry was cast on Ni foam. A platinum foil and a saturated calomel electrode (SCE) were used as the counter and reference electrode, respectively. The strong electrolyte, $0.5 \mathrm{M} \mathrm{Na}_{2} \mathrm{SO}_{4}$ solution, was used to ensure high ionic strength. Cyclic voltammetry was performed in the voltage range of $-0.6 \mathrm{~V}$ to $0.4 \mathrm{~V}$ at scan rates of 1,5 or $10 \mathrm{mVs}-1$, respectively. Galvanostatic charge/discharge experiments were carried out in the potential range from $-600 \mathrm{mV}$ to $400 \mathrm{mV}$ with an applied current density of $200,400,800$ or $1000 \mathrm{~mA} / \mathrm{g}$, respectively.

\section{RESULTS AND DISCUSSION}

\section{A. Morphology Analysis}

Images showed that the SFC surface (Figure 1a) was the smooth. This observation clearly indicated that pretreatment could remove the hemicellulose and lignin coverings from the SFC surfaces. In SFC/PPy composites, the presence of PPy particles coated on SFC can be observed (Figure $1 \mathrm{c}-\mathrm{d}$ ). The thickness layer of polypyrrole deposited on SFC increased and the PPy particles tend to agglomerate with increasing pyrrole concentration. Apparently, a uniform coating layer was achieved for SFC/PPy composites prepared with 30wt $\%$ and 50wt\% SFC. As the SFC content increased, a more homogeneous coating was formed. From SEM observations, it is possible to state that the initial content of SFC determines the thickness of the PPy layer defines the final microstructure and coating quality.

\section{B. FTIR Spectroscopy}

The FTIR spectra of SFC and SFC/PPy composites are shown in Figure 2. The characteristic IR peaks of SFC includes a broad band at $3403 \mathrm{~cm}^{-1}$ attributed to $\mathrm{O}-\mathrm{H}$ stretching vibration, a band at $2897 \mathrm{~cm}^{-1}$ assigned to aliphatic C-H stretching vibration. The SFC/PPy composites exhibited a band at 3429 $\mathrm{cm}^{-1}$ for $\mathrm{N}-\mathrm{H}$ stretching vibration instead of $3403 \mathrm{~cm}^{-1}$ for $\mathrm{O}-\mathrm{H}$ stretching, which results from the complete wrapping of PPy around SFC fibers. Similar quenching of aliphatic C-H stretching at $2897 \mathrm{~cm}^{-1}$ was also observed. Moreover, the characteristic peaks for PPy were identical to those for SFC/PPy composites, which include a band at $1542 \mathrm{~cm}^{-1}$ assigned to the $\mathrm{C}=\mathrm{C}$ stretching vibration in the Py ring, band at $1459 \mathrm{~cm}^{-1}$ corresponding to the $\mathrm{C}=\mathrm{N}$ stretching vibration in the $\mathrm{Py}$ ring [2]. Moreover, the absorbance of $\mathrm{S}=\mathrm{O}$ stretching at $1180 \mathrm{~cm}^{-1}$ of sulfonate, indicated that PPy had been doped with sodium p-toluene sulfonate.

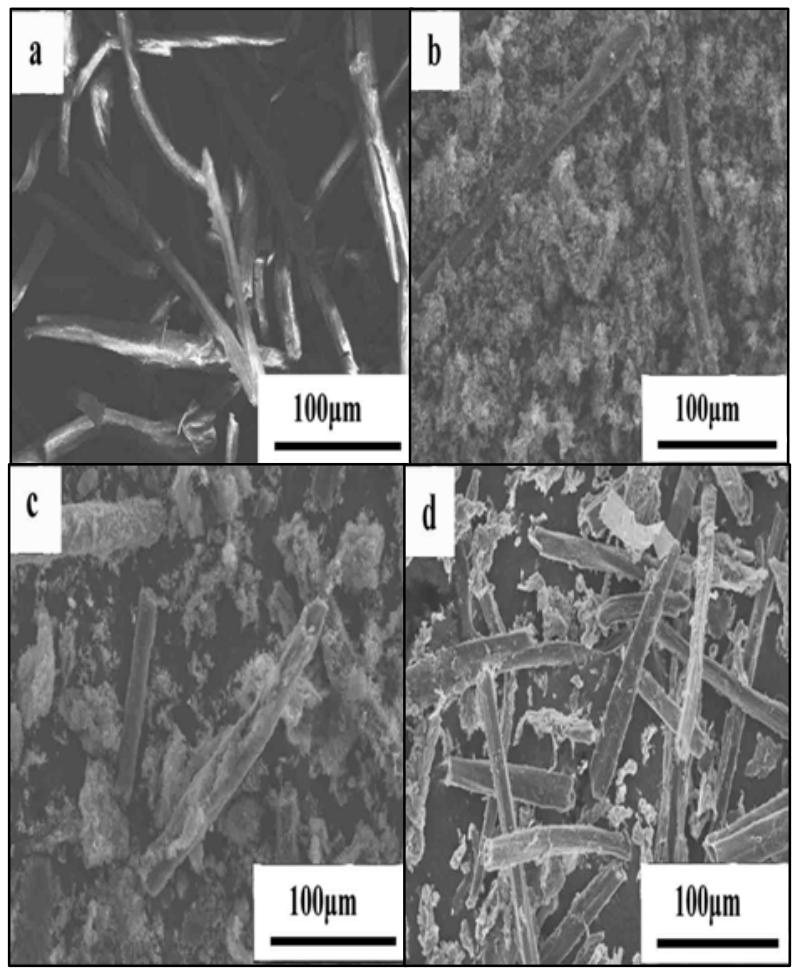

FIGURE I. SEM OF SFC AND SFC/ PPY COMPOSITES (A: SFC; B: S1; C: S3; D: S5).

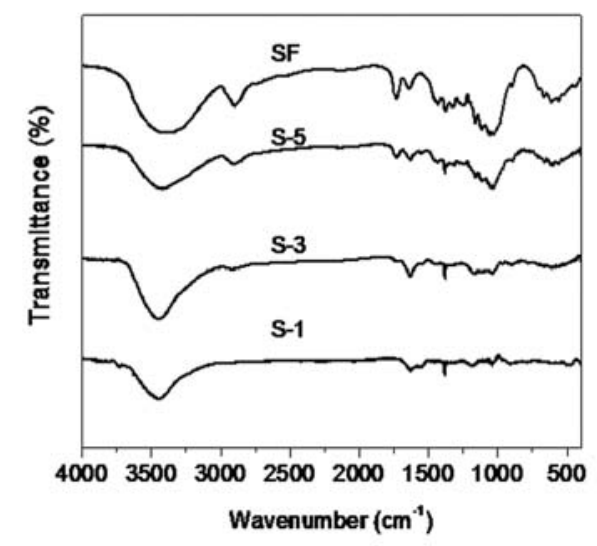

FIGURE II. FTIR SPECTRA OF SFC AND SFC/PPY COMPOSITES. 


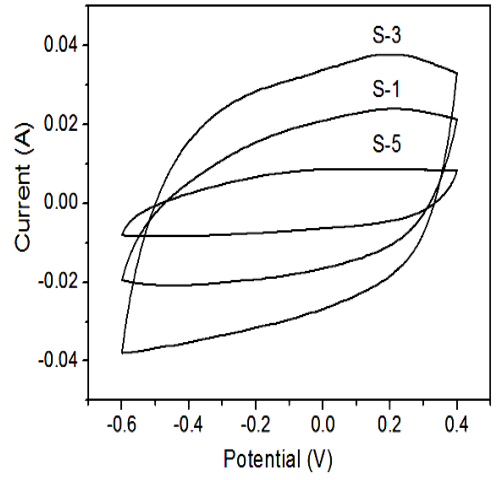

FIGURE III. CV CURVES OF SFC/PPY COMPOSITES

\section{Electrochemical Performances}

The potential applications of as-prepared SFC/PPy nanocomposites were explored by fabricating the samples into supercapacitor electrodes and characterizing with cyclic voltammograms (CVs), and galvanostatic charge/discharge measurements. CVs response of SFC/PPy carried out at varied scan rates between -0.6 and $+0.4 \mathrm{~V}$ is shown in Figure 3 .

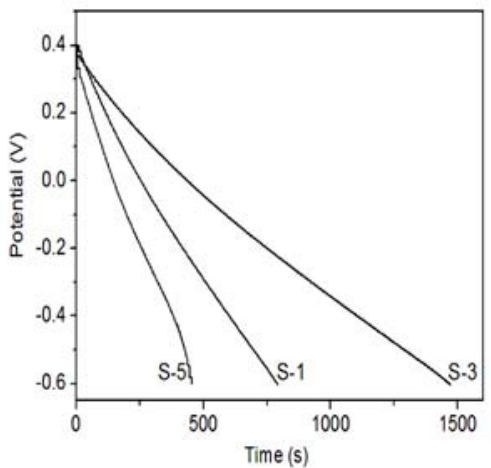

FIGURE IV. DISCHARGE CURVES OF SFC/PPY COMPOSITES(0.2A/G).

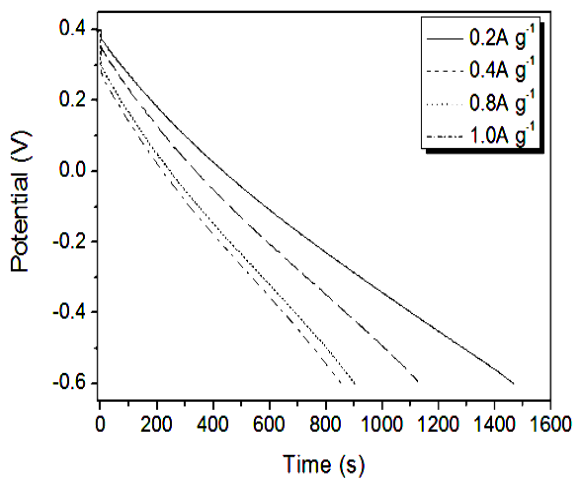

FIGURE V. DISCHARGE CURVES OF S3.

The composite showed a high degree of electroactivity, with the rectangular $\mathrm{CV}$ traces showing the transitions from reduced to oxidized forms, which demonstrates the retention of the important redox feature of conducting polymers in the as-synthesized SFC/PPy nanocomposites .
As illustrated in Figure 4, during the charge and discharge process, the charge curve of SFC/PPy is almost symmetric to its corresponding discharge counterpart with a slight curvature, indicating the pseudo capacitance behavior. The Cs values of SFC/PPy composites were calculated to be 195, 244, 367, 187, $114 \mathrm{~F} / \mathrm{g}$ at current densities of $200 \mathrm{~mA} / \mathrm{g}$, respectively. According to the literature [7], the cellulose exhibited only negligible electroactivity. Galvanostatic discharge for S-3 sample was 367, 281, 227, $212 \mathrm{~F} / \mathrm{g}$ performed at current densities of 200, 400, 800 and $1000 \mathrm{~mA} / \mathrm{g}$. About $57.8 \%$ of Cs was retained when the current density increased five times, which was attributed to the discrepant insertion-disinsertion behavior of alkali ion from the electrode to PPy.

\section{SUMMARY}

It has been shown that it is possible to manufacture an electrical conducting composite material composed of natural cellulose and polypyrrole by direct chemical polymerization of pyrrole on sisal microcrystalline cellulose without the need for sophisticated techniques. The excellent performances of SFC/PPy composites pave the way toward promising applications in various electronic devices.

\section{ACKNOWLEDGEMENTS}

This work was supported by the National Natural Science Foundation of China (51303035, 21264005), Guangxi Natural Science Foundation (2013GXNSFBA019041), Training Programs of Innovation and Entrepreneurship for Undergraduates, the Guangxi Funds for a Specially-Appointed Expert, and the Guangxi Small Highland Innovation Team of Talents in Colleges and Universities. The correesponding authors are Chao Yang and Limin Zang.

\section{REFERENCES}

[1] M.M. Khin, A.S. Nair, V.J. Babu, R. Murugan, S. Ramakrishna, A review on nanomaterials for environmental remediation, Energy Environ. Sci. 5 (2012) 8075-8109.

[2] C. Yang, L.M. Zang, J.H. Qiu, E. Sakai, X.L. Wu, Y.K. Iwase, Nano-cladding of natural microcrystalline cellulose with conducting polymer: preparation, characterization, and application in energy storage, RSC Adv. 4 (2014) 40345-40351.

[3] H. Wang, L. Bian, P. Zhou, J. Tang, W. Tang, Core-sheath structured bacterial cellulose/polypyrrole nanocomposites with excellent conductivity as supercapacitors, J. Mater. Chem. A 1 (2013) 578-584.

[4] C. Yang, H.M. Mo, L.M. Zang, J.H. Qiu, H. You, X. Yang, Structural investigation of anionic functional poly(vinyl alcohol) doped polypyrrole nanospheres, Fiber. Polym. 15 (2014) 2019-2025.

[5] C. Yang, H. Mo, L. Zang, J. Qiu, E. Sakai, X. Wu, A facile method to synthesize polypyrrole nanoparticles in the presence of natural organic phosphate, Physica B 449 (2014)181-185.

[6] C. Yang, H.M. Mo, L.M. Zang, J.H. Qiu, X. Wang, H. You, Preparation and characterization of coaxial mullite/polypyrrole fibrous nanocomposites via self-assembling and in situ surface-initiated polymerization, Polym. Compos. 35 (2014) 892-899.

[7] L.M. Zang, J.H. Qiu, C. Yang,E. Sakai,Enhanced conductivity and electrochemical performance of electrode material based on multifunctional dye doped polypyrrole, J. Nanosci. Nanotechnol. In press (doi:10.1166/jnn.2015.10759). 